\title{
Impact of Implementation of Corporate Governance Guidelines (Clause 49) on Financial Performance of Indian Listed firms
}

\author{
Effulgence \\ Vol. 15 No. 2 \\ July-December, 2017 \\ Rukmini Devi Institute of Advanced Studies \\ E-mail : effulgence@rdias.ac.in, Website : www.rdias.ac.in \\ http:/ / effulgence.rdias.ac.in/user/default.aspx \\ https://dx.doi.org/10.33601/effulgence.rdias/v15/i2/2017/1-16
}

\section{Dr. Prashant Gupta ${ }^{1}$ \\ Dr. Dinesh Kumar Sharma ${ }^{2}$}

\begin{abstract}
Generally the good corporate governance reduces the agency problem and improves the firm/enterprise's performance. It not only cubs the corporate frauds, scandals etc, but also improves the corporate image in the minds of all stakeholders and public at large. During liberalization in early nineties, evolution of corporate governance also started in India from Cadbury Committee Report to Kumar Manglam Birla report on Corporate Governance. Then, came the formal code of conduct or guidelines issued by the regulator of capital markets, Securities Exchange Board of India (SEBI) by inserting a new 'Clause 49' in the listing guidelines to make these mandatory for all the listed companies in India from January, 2006. The question is to determine how governance and firm performance interact with each other and study their relationship to find some meaningful results. This study examined the impact of the implementation of the Clause 49 (corporate governance) on the firm's performance in India. Since it is made compulsory for the listed companies, hence this study is done on the companies listed in the NSE. Both market and accounting based performance measures are used for the purpose. Unique data from 50 listed firms drawn from the index "Nifty 50" to represent the Indian listed firms for 16 years, covering eight years period each for prior and after 2006 to find out the impact by comparing the results.
\end{abstract}

The result of the study suggests that there is no positive impact of implementation of Clause 49 on the performance of the Indian listed companies.

Keywords: Corporate Governance, Clause 49 and Firm Performance

\section{INTRODUCTION}

Q orporate governance empowers the board so that it can perform its duties being unbiased to promoter shareholders and/or to the top management of the firm, i.e. to resolve the agency problem and protect the shareholder's benefits at large. Hence, the corporate governance is the process, rule and/or the laws to supervise, manage and govern the firms without under the undue influence of any one and to serve the all stakeholders. So, corporate governance mitigates the agency problems arising due to the complex relationship between agents and principals.

1. IMI, New Delhi, prashantgupta@imi.edu

2. Gautam Budh University, Greater Noida, dinesh@gbu.ac.in

Corresponding Author

Dr. Dinesh Kumar Sharma, Gautam Budh University, Greater Noida, dinesh@gbu.ac.in 
Then what the Corporate Governance is all about? It is a relevant set of mechanisms to economic efficiency that has influence over investor's decision to provide finance to firm; debt or equity (Shleifer and Vishny, 1997). Mayer (1997) defined corporate governance as ways of bringing investor's and manager's interest in line to ensure firm's functioning for the investor's benefit. Corporate governance includes the structures, processes, cultures and systems that create organizations' successful operation (Keasey et al., 1997). Significant flow of capital to the financing of firms can be ensured with governance structure. Information asymmetry between suppliers' of capital and managers can cause into expropriation of investor's wealth due to separation between control and ownership (Berle and Means, 1932). This could be dealt properly with an appropriate governance structure in place that should take care of reducing rent seeking behavior of an agent while providing required necessary investment. As described by Deakin and Hughes (1997), corporate governance is the relationship between conception of society about the scope of firm's accountability and the mechanism adopted by the firm for internal governance. Due to presence of agency problem, for future decisions and any unforeseen situation that is not described in the current agreement between principal and agent, it is necessary to have a mechanism for this. Hart (1996) described that structure of governance provide a way to decide those actions which may be taken in future but were not initially specified in the contract. From the above discussion we can deduce that the structure and process set up by a company or enterprise to reduce the agency problem as ownership and controls are separated. Various different systems of corporate governance will draw a clear structure and legitimate accountability by explaining relationship between company and its key functionaries.

Due to East Asian crisis, increasing integration of business across the globe and some corporate scandals/frauds around the world, prominence and impetus to corporate governance has increased world over. The existing literature on corporate governance is generally on and about large and listed firms in the US and UK, and these studies primarily focused on the relationship between corporate performance, structures of ownership and composition of boards.

In many Asian countries like India, Japan, Korea, and also in some South-East Asian countries large number of businesses are managed and owned by business families. By using cross investment and stock pyramids, these families retain their control on the businesses even without a controlling stake. A divergence between cash flow rights and voting (or control) rights is created due to this.

In Indian context, generally the agency problem is at the core of corporate governance, which is a conflict between shareholders and management. Earlier, companies who were going global started following stringent policies, disclosures and international accounting standards, but now after clause 49 for all listed companies and with new company law in place, most others are now considering it necessary to do so since they have to follow the current norms. In India, the Anglo-American model is generally followed that lays more importance to shareholders as compared to stakeholders.

The major areas of compliance in Clause 49 are :

- Enhanced role of Audit committee

- $\mathrm{CEO} / \mathrm{CFO}$ Certification of Accounts by $\mathrm{CEO} / \mathrm{CFO}$

- Appointment of Independent Directors as per the requirements

- Code of Conduct for Board / Senior Management

- Legal Compliance Report and Risk Mitigation Report to the Board

- Compliance, related to Subsidiary Companies

- Information items to Board

\section{Review of Literature:}

Diverse, rather many times contrast objectives 
emerge between owners and managers of business. This requires a system to control the cost associated with this divergence. Corporate governance serves that purpose and agency theory dominates the corporate governance literature.

It is generally accepted that there is a positive effect of good corporate governance on performance of firms that is enhanced due to good governance. Several studies supported this view (Weisbach, 1988; Brickley and James, 1987; Rosenstein and Wyatt, 1990; Lee et al, 1992; Byrd and Hickman, 1992; Brickley et al, 1994; Hossain et al, 2000; Chung et al, 2003). On the contrary to this generally accepted notion about positive impact of good governance on firm performance, many studies have found that there is no or negative relationship between performance of firm and good corporate governance (Prevost et al. 2002; Park and Shin, 2003; Young, 2003; Singh and Davidson, 2003; Bathala and Rao, 1995; Hutchinson, 2002) or have not found any relationship.

Some studies tried to find out the reasons of these diverse results and inconsistencies. Some found it is due to the limited scope of sources being it a survey data or publicly available data. Krivogorsky (2006) argued that the literature in corporate governance generally use only two variables at a time. For example, studies by McAvoy et al. (1983) and Hermalin and Weisbach (1991) studied relationship between firm performance and composition of board and Demsetz and Villalonga (2001) analysed the correlation between firm performance and managerial ownership. Furthermore, Gani and Jermias, (2006) pointed out that the restrictive use of accounting based measures like return on equity (ROE), return on assets (ROA) and return on capital employed (ROCE) or market based measures like market value of equities may be the reason of these inconsistencies.

According to Shleifer and Vishny (1997) in their extensive literature survey on corporate governance, suggested that shareholder's and creditor's control over managers can be minimized by incorporating effective corporate governance. Jensen and Meckling (1976) have discussed about relationship between shareholders and managers and suggested that since managers mange the company on behalf of shareholders so managers are agents of shareholders and thus they must judiciously utilize the financial resources supplied by shareholders to the business. Now days, a lot is debated and discussed about the board's functioning and structures so this has become an important issue to be monitored by legal/regulatory authorities and also subject to continuous scrutiny by media. Quality of manager's decision making can be enhanced and improved by better board supervision (Monks and Minow, 1995). The association between board size and financial performance was analyzed on the data of 504 firms drawn from 18 industries by Kathuria and Dash (1999) in India. They found that if firm size grows, contribution of additional board member decrease if board size is increased but with increase in board size, performance of firm increases.

Another study, done by Dhawan (2006) in India on the large listed firms to identify the role of the directors (Board) in the corporate governance practices, found that the size of the board increases with the increase in turnover initially but after a certain level, increase in turnover has no influence on board size. He also found that what matter more than the size of board is, the appropriate combination of knowledge and skills. Relationship between Board parameters and financial performance of firms is empirically examined by Ghosh (2006) on 127 listed manufacturing firms and it was found that irrespective of market based or accounting based performance measures, there is negative influence of board size on the performance of firm. Also it is found that there is a significant impact of Chief Executive Officer's compensation on performance of firm. Generally it is believed that independence of board leads to better firm performance. A study based on factor analysis is done by Prasanna (2006) that empirically proved this belief. The study suggested that the independent 
directors effectively lead various committees formed for better governance, make board more credible for improved governance and they also help board in functioning more efficiently and effectively. In an another study done by Mayur and Saravanan (2006), who studied the relationship between performance of Indian banks and three chosen board parameters, it is deducted that board size does not affect the value of Bank. Later SEBI also came out with Clause 49 for all the listed companies that emphasized the importance and role of independent directors for the enhanced transparency and improved governance as compared to only executive/functional directors in the board. Recommendations by other committees like Kumar Manglam Birla Committee along with other recommendations also argued and advocated for independency of board for effective governance structure. Hence composition of board can be considered one of the important parameter in relation to performance of firm. Kumar and Singh (2012) conducted a study in 2008 on 157 nonfinancial Indian companies to find out the effectiveness of outside directors in boards of companies. The results of this study do not strongly confirm that more independent directors in board have positive impact on firm's value. On the other hand a study, done by Jackling and Johl (2009) on Indian companies supported agency theory and found that firm performance improves if more independent directors are inducted in boards of companies. Results of this study also opposed the idea of disassociation of ownership and control i.e. a strong owner cum (duality role, CEO is the promoter, and also the only board manager) have a negative impact on firm performance was not supported by this study. So the various studies which examined between firm performance and internal ownership can be categorized in to two sets; one with positive relationship and another with negative relationship between the two aspects. Impact of insider ownership on firm performance is industry specific and not general (Phani et al., 2005). This study examined the difference in cash flow rights and insiders control and also its impact on the individual firm performance. In a study on the relationship between firm value and independence of audit committee by Chan and $\mathrm{Li}$ (2008) on fortune 200 companies, it is found that if top executives of other publically listed companies are considered as 'expert independent directors' then their presence in board or in audit committee as 'expert independent directors', increases the value of firm. The audit committee size has significant effect on the value of firm, however independence of audit committee and no. of meetings of audit committee were found insignificant with firm performance in a study conducted by Al-Matari et.al.(2012) on listed companies of Saudi on Saudi stock exchange (TADWAL) in year 2010. An increasing trend towards the compliance of clause 49 of SEBI was observed in the listed companies in a content analysis, done by Bhasin (2012) on top 500 listed companies in India.

\section{Rationale of the study}

Literature review suggests that lots of studies have been done on to find out the impact of corporate governance on firm performance. Many researchers have studied different aspects of corporate governance and linked these to firm performance like Board Size, Number of Independent Directors in board, Audit committees meetings etc. But we found very fewer studies find out the performance of Indian listed companies after the implementation of Clause 49 by the SEBI in 2006. So the researchers thought to study this impact on the Indian listed companies.

\section{Objectives of the study}

The objective of the study is:

- To find out the impact of implementation of Corporate Governance Guidelines (Clause 49) on performance of listed companies in India. To find out the impact on firm performance, various profitability parameters are tested (like EPS, leverage, gross profit and net profit etc.) of listed companies. 


\section{Research Methodology}

The research methodology is based on the secondary source of information. In order to evaluate the financial performance and impact of implementation of clause 49 on performance of listed companies in India, various alternative hypotheses has been formulated which are tested through t-test (paired two samples for means). The present study covers listed firms drawn from the index "Nifty 50" to represent the Indian listed firms for a period of 16 years, which shows eight years study prior and eight years study after the implementation of the Clause 49 , the corporate governance guidelines by the SEBI in 2006. Researchers have taken the current list of Nifty 50 companies, which may not be the same for the entire 16 years of study as some companies keep on changing in the list from time to time. So, data of the companies mentioned in the current list of Nifty 50 index is taken for this research purpose to maintain the consistency from prowess, moneycontrol.com and other sources. All data has been collected and compiled judiciously for this study.

\section{Research Hypotheses:}

The research hypotheses are formulated keeping the above objectives of the study in the mind. The following are the alternative hypotheses tested during the study period only.
H1: There is significance difference in the earning per share of Nifty-50 companies between pre and post implementation of clause- 49 of corporate governance in India.

H2: There is significance difference in the gross profit ratio of Nifty-50 companies between pre and post implementation of clause- 49 of corporate governance in India.

H3: There is significance difference in the net profit ratio of Nifty-50 companies between pre and post implementation of clause- 49 of corporate governance in India.

H4: There is significance difference in the financial leverage of Nifty-50 companies between pre and post implementation of clause- 49 of corporate governance in India.

\section{Analysis and Discussion:}

In this study alternate hypotheses are tested with the help of $t$-test (paired two samples for means) at $5 \%$ level of significance.

\section{Hypothesis 1:}

H1: There is significance difference in the earning per share of Nifty-50 companies between pre and post implementation of clause- 49 of corporate governance in India.

\begin{tabular}{|l|l|r|r|r|r|r|r|}
\hline \multicolumn{2}{|l}{} & \multicolumn{7}{|c|}{ Table-1 Paired Samples Test } & \multirow{2}{*}{$\begin{array}{c}\text { Sig. } \\
\text { t-value }\end{array}$} & $\begin{array}{c}\text { Paired Differences } \\
\text { (2-tailed) }\end{array}$ & Result of Hypotheses \\
\cline { 2 - 7 } & Mean & Std. Deviation & Std. Error Mean & & \\
\hline Pair 1 & $\begin{array}{l}\text { Pre-EPS A C C Ltd. - } \\
\text { Post- EPS A C C Ltd. }\end{array}$ & -52.080 & 20.066 & 7.094 & -7.341 & 0.000 & Accepted \\
\hline Pair 2 & $\begin{array}{l}\text { Pre-EPS Adani Ports and Special } \\
\text { Economic Zone Ltd. - Post- EPS Adani } \\
\text { Ports and Special Economic Zone Ltd. }\end{array}$ & -7.589 & 4.580 & 1.619 & -4.686 & 0.002 & Accepted \\
\hline Pair 3 & $\begin{array}{l}\text { Pre-EPS Ambuja Cements Ltd. - } \\
\text { Post- EPS Ambuja Cements Ltd. }\end{array}$ & -21.360 & 7.725 & 2.731 & -7.821 & 0.000 & Accepted \\
\hline Pair 4 & $\begin{array}{l}\text { Pre-EPS Asian Paints Ltd. - } \\
\text { Post- EPS Asian Paints Ltd. }\end{array}$ & -53.986 & 37.019 & 13.088 & -4.125 & 0.004 & Accepted \\
\hline
\end{tabular}


ISSN (O) : 2456-6675, ISSN (P) : 0972-8058

\begin{tabular}{|c|c|c|c|c|c|c|c|}
\hline Pair 5 & $\begin{array}{l}\text { Pre-EPS Axis Bank Ltd.- } \\
\text { Post- EPS Axis Bank Ltd. }\end{array}$ & -59.356 & 31.223 & 11.039 & -5.377 & 0.001 & Accepted \\
\hline Pair 6 & $\begin{array}{l}\text { Pre-EPS Bajaj Auto Ltd.- } \\
\text { Post-EPS Bajaj Auto Ltd. }\end{array}$ & -9.551 & 29.998 & 10.606 & -0.901 & 0.398 & Rejected \\
\hline Pair 7 & $\begin{array}{l}\text { Pre-EPS Bank Of Baroda - } \\
\text { Post- EPS Bank Of Baroda }\end{array}$ & -59.218 & 31.532 & 11.148 & -5.312 & 0.001 & Accepted \\
\hline Pair 8 & $\begin{array}{l}\text { Pre-EPS Bharat Heavy Electricals Ltd. - } \\
\text { Post- EPS Bharat Heavy Electricals Ltd. }\end{array}$ & -60.383 & 39.241 & 13.874 & -4.352 & 0.003 & Accepted \\
\hline Pair 9 & $\begin{array}{l}\text { Pre-EPS Bharat Petroleum Corpn. Ltd. - } \\
\text { Post- EPS Bharat Petroleum Corpn. Ltd. }\end{array}$ & 5.081 & 49.320 & 17.437 & 0.291 & 0.779 & Rejected \\
\hline Pair 10 & $\begin{array}{l}\text { Pre-EPS BhartiAirtel Ltd. - Post- EPS } \\
\text { BhartiAirtel Ltd. }\end{array}$ & -32.201 & 10.944 & 3.869 & -8.322 & 0.000 & Accepted \\
\hline Pair 11 & $\begin{array}{l}\text { Pre-EPS Bosch Ltd. - Post- EPS Bosch } \\
\text { Ltd. }\end{array}$ & -163.488 & 57.670 & 20.390 & -8.018 & 0.000 & Accepted \\
\hline Pair 12 & $\begin{array}{l}\text { Pre-EPS Cairn India Ltd. - Post-EPS } \\
\text { Cairn India Ltd. }\end{array}$ & -14.339 & 28.894 & 10.216 & -1.404 & 0.203 & Rejected \\
\hline Pair 13 & Pre-EPS Cipla Ltd. - Post- EPS Cipla Ltd. & -9.773 & 16.615 & 5.874 & -1.664 & 0.140 & Rejected \\
\hline Pair 14 & $\begin{array}{l}\text { Pre-EPS Coal India Ltd. - Post- EPS Coal } \\
\text { India Ltd. }\end{array}$ & -18.745 & 11.005 & 3.891 & -4.818 & 0.002 & Accepted \\
\hline Pair 15 & $\begin{array}{l}\text { Pre-EPS Dr. Reddy'S Laboratories Ltd. - } \\
\text { Post- EPS Dr. Reddy'S Laboratories Ltd. }\end{array}$ & -54.596 & 68.555 & 24.238 & -2.253 & 0.059 & Rejected \\
\hline Pair 16 & $\begin{array}{l}\text { Pre-EPS G A I L (India) Ltd. - Post- EPS } \\
\text { G A I L (India) Ltd. }\end{array}$ & -10.514 & 4.909 & 1.736 & -6.058 & 0.001 & Accepted \\
\hline Pair 17 & $\begin{array}{l}\text { Pre-EPS Grasim Industries Ltd. - Post- } \\
\text { EPS Grasim Industries Ltd. }\end{array}$ & -99.778 & 69.736 & 24.655 & -4.047 & 0.005 & Accepted \\
\hline Pair 18 & $\begin{array}{l}\text { Pre-EPS H C L Technologies Ltd. - Post- } \\
\text { EPS H C L Technologies Ltd. }\end{array}$ & -74.284 & 97.551 & 34.489 & -2.154 & 0.068 & Rejected \\
\hline Pair 19 & $\begin{array}{l}\text { Pre-EPS H D F C Bank Ltd. - Post- EPS H } \\
\text { D F C Bank Ltd. }\end{array}$ & -68.711 & 33.709 & 11.918 & -5.765 & 0.001 & Accepted \\
\hline Pair 20 & $\begin{array}{l}\text { Pre-EPS Hero Motocorp Ltd. - Post- EPS } \\
\text { Hero Motocorp Ltd. }\end{array}$ & -312.403 & 94.733 & 33.493 & -9.327 & 0.000 & Accepted \\
\hline Pair 21 & $\begin{array}{l}\text { Pre-EPS Hindalco Industries Ltd. - Post- } \\
\text { EPS Hindalco Industries Ltd. }\end{array}$ & -29.425 & 64.427 & 22.778 & -1.292 & 0.237 & Rejected \\
\hline Pair 22 & $\begin{array}{l}\text { Pre-EPS Hindustan Unilever Ltd. - Post- } \\
\text { EPS Hindustan Unilever Ltd. }\end{array}$ & 56.888 & 203.813 & 72.059 & 0.789 & 0.456 & Rejected \\
\hline Pair 23 & $\begin{array}{l}\text { Pre-EPS Housing Development Finance } \\
\text { Corpn. Ltd. - Post- EPS Housing } \\
\text { Development Finance Corpn. Ltd. }\end{array}$ & -43.821 & 112.816 & 39.886 & -1.099 & 0.308 & Rejected \\
\hline Pair 24 & $\begin{array}{l}\text { Pre-EPS I C I C I Bank Ltd. - Post- EPS I } \\
\text { C I C I Bank Ltd. }\end{array}$ & -33.918 & 11.437 & 4.044 & -8.388 & 0.000 & Accepted \\
\hline Pair 25 & Pre-EPS I T C Ltd. - Post- EPS I T C Ltd. & -37.465 & 19.062 & 6.739 & -5.559 & 0.001 & Accepted \\
\hline Pair 26 & $\begin{array}{l}\text { Pre-EPS Idea Cellular Ltd. - Post- EPS } \\
\text { Idea Cellular Ltd. }\end{array}$ & -2.751 & 1.207 & 0.427 & -6.446 & 0.000 & Accepted \\
\hline Pair 27 & $\begin{array}{l}\text { Pre-EPS Indusind Bank Ltd. - Post- EPS } \\
\text { Indusind Bank Ltd. }\end{array}$ & -5.860 & 10.197 & 3.605 & -1.625 & 0.148 & Rejected \\
\hline Pair 28 & $\begin{array}{l}\text { Pre-EPS Infosys Ltd. - Post- EPS Infosys } \\
\text { Ltd. }\end{array}$ & -35.450 & 109.255 & 38.627 & -0.918 & 0.389 & Rejected \\
\hline Pair 29 & $\begin{array}{l}\text { Pre-EPS Kotak Mahindra Bank Ltd. - } \\
\text { Post- EPS Kotak Mahindra Bank Ltd. }\end{array}$ & -10.704 & 14.118 & 4.991 & -2.144 & 0.069 & Rejected \\
\hline Pair 30 & $\begin{array}{l}\text { Pre-EPS Larsen \& Toubro Ltd. - Post- } \\
\text { EPS Larsen \& Toubro Ltd. }\end{array}$ & -212.603 & 141.166 & 49.910 & -4.260 & 0.004 & Accepted \\
\hline Pair 31 & $\begin{array}{l}\text { Pre-EPS Lupin Ltd. - Post- EPS Lupin } \\
\text { Ltd. }\end{array}$ & -79.399 & 63.546 & 22.467 & -3.534 & 0.010 & Accepted \\
\hline Pair 32 & $\begin{array}{l}\text { Pre-EPS Mahindra \& Mahindra Ltd. - } \\
\text { Post- EPS Mahindra \& Mahindra Ltd. }\end{array}$ & -48.790 & 30.819 & 10.896 & -4.478 & 0.003 & Accepted \\
\hline Pair 33 & $\begin{array}{l}\text { Pre-EPS Maruti Suzuki India Ltd. - Post- } \\
\text { EPS Maruti Suzuki India Ltd. }\end{array}$ & -107.123 & 38.197 & 13.505 & -7.932 & 0.000 & Accepted \\
\hline
\end{tabular}


ISSN (O) : 2456-6675, ISSN (P) : 0972-8058

\begin{tabular}{|c|c|c|c|c|c|c|c|}
\hline Pair 34 & $\begin{array}{l}\text { Pre-EPS N T P C Ltd. - Post- EPS N T P } \\
\text { C Ltd. }\end{array}$ & -4.681 & 2.097 & 0.742 & -6.313 & 0.000 & Accepted \\
\hline Pair 35 & $\begin{array}{l}\text { Pre-EPS Oil \& Natural Gas Corpn. Ltd. - } \\
\text { Post- EPS Oil \& Natural Gas Corpn. Ltd. }\end{array}$ & -4.900 & 43.739 & 15.464 & -0.317 & 0.761 & Rejected \\
\hline Pair 36 & $\begin{array}{l}\text { Pre-EPS Power Grid Corpn. Of India } \\
\text { Ltd. - Post- EPS Power Grid Corpn. Of } \\
\text { India Ltd. }\end{array}$ & -3.671 & 1.682 & 0.595 & -6.175 & 0.000 & Accepted \\
\hline Pair 37 & $\begin{array}{l}\text { Pre-EPS Punjab National Bank - Post- } \\
\text { EPS Punjab National Bank }\end{array}$ & -73.353 & 32.446 & 11.472 & -6.394 & 0.000 & Accepted \\
\hline Pair 38 & $\begin{array}{l}\text { Pre-EPS Reliance Industries Ltd. - Post- } \\
\text { EPS Reliance Industries Ltd. }\end{array}$ & -43.013 & 36.950 & 13.064 & -3.292 & 0.013 & Accepted \\
\hline Pair 39 & $\begin{array}{l}\text { Pre-EPS State Bank Of India - Post- EPS } \\
\text { State Bank Of India }\end{array}$ & -75.629 & 38.759 & 13.704 & -5.519 & 0.001 & Accepted \\
\hline Pair 40 & $\begin{array}{l}\text { Pre-EPS Sun Pharmaceutical Inds. Ltd. - } \\
\text { Post- EPS Sun Pharmaceutical Inds. Ltd. }\end{array}$ & -65.450 & 55.162 & 19.503 & -3.356 & 0.012 & Accepted \\
\hline Pair 41 & $\begin{array}{l}\text { Pre-EPS Tata Consultancy Services Ltd. - } \\
\text { Post- EPS Tata Consultancy Services Ltd. }\end{array}$ & -401.091 & 95.904 & 33.907 & -11.829 & 0.000 & Accepted \\
\hline Pair 42 & $\begin{array}{l}\text { Pre-EPS Tata Motors Ltd. - Post- EPS } \\
\text { Tata Motors Ltd. }\end{array}$ & -21.045 & 34.709 & 12.271 & -1.715 & 0.130 & Rejected \\
\hline Pair 43 & $\begin{array}{l}\text { Pre-EPS Tata Power Co. Ltd. - Post- EPS } \\
\text { Tata Power Co. Ltd. }\end{array}$ & -14.293 & 9.237 & 3.266 & -4.376 & 0.003 & Accepted \\
\hline Pair 44 & $\begin{array}{l}\text { Pre-EPS Tata Steel Ltd. - Post- EPS Tata } \\
\text { Steel Ltd. }\end{array}$ & -36.511 & 25.100 & 8.874 & -4.114 & 0.004 & Accepted \\
\hline Pair 45 & $\begin{array}{l}\text { Pre-EPS Tech Mahindra Ltd. - Post- EPS } \\
\text { Tech Mahindra Ltd. }\end{array}$ & -4.691 & 20.848 & 7.371 & -0.636 & 0.545 & Rejected \\
\hline Pair 46 & $\begin{array}{l}\text { Pre-EPS Ultratech Cement Ltd. - Post- } \\
\text { EPS Ultratech Cement Ltd. }\end{array}$ & -70.855 & 18.919 & 6.689 & -10.593 & 0.000 & Accepted \\
\hline Pair 47 & $\begin{array}{l}\text { Pre-EPS Vedanta Ltd. - Post- EPS } \\
\text { Vedanta Ltd. }\end{array}$ & -178.984 & 184.929 & 65.382 & -2.737 & 0.029 & Accepted \\
\hline Pair 48 & $\begin{array}{l}\text { Pre-EPS Wipro Ltd. - Post- EPS Wipro } \\
\text { Ltd. }\end{array}$ & 24.444 & 62.900 & 22.239 & 1.099 & 0.308 & Rejected \\
\hline Pair 49 & $\begin{array}{l}\text { Pre-EPS Yes Bank Ltd. - Post- EPS Yes } \\
\text { Bank Ltd. }\end{array}$ & -19.645 & 13.904 & 4.916 & -3.996 & 0.005 & Accepted \\
\hline Pair 50 & $\begin{array}{l}\text { Pre-EPS Zee Entertainment Enterprises } \\
\text { Ltd. - Post- EPS Zee Entertainment } \\
\text { Enterprises Ltd. }\end{array}$ & -35.001 & 29.010 & 10.257 & -3.413 & 0.011 & Accepted \\
\hline
\end{tabular}

The results of Paired Samples t-Test in Table-1 depict that the alternative hypotheses of 16 companies have been rejected while it has been accepted for 34 companies. Hence statistically there is significance impact of implementation of clause- 49 of corporate governance on the shareholder's wealth of majority of nifty-50 companies in India. The industries were further clustered on the basis of acceptance and rejection of the relevant hypotheses. It has been observed that most of the bank's EPS have been impacted significantly.

\section{Hypothesis 2:}

H2: There is significance difference in the gross profit ratio of Nifty-50 companies between pre and post implementation of clause- 49 of corporate governance in India. 


\begin{tabular}{|c|c|c|c|c|c|c|c|}
\hline \multicolumn{8}{|c|}{ Table-2 Paired Samples Test } \\
\hline & & \multicolumn{3}{|c|}{ Paired Differences } & \multirow[b]{2}{*}{ t-value } & \multirow{2}{*}{$\begin{array}{c}\text { Sig. } \\
\text { (2-tailed) }\end{array}$} & \multirow{2}{*}{$\begin{array}{c}\text { Result of } \\
\text { Hypothese } \\
\text { s }\end{array}$} \\
\hline & & Mean & $\begin{array}{c}\text { Std. } \\
\text { Deviation }\end{array}$ & $\begin{array}{l}\text { Std. Error } \\
\text { Mean }\end{array}$ & & & \\
\hline Pair 1 & Pre-GP Ratio A C C Ltd. - Post- GP Ratio A C C Ltd. & -8.767 & 11.705 & 4.424 & -1.982 & 0.095 & Rejected \\
\hline Pair 2 & $\begin{array}{l}\text { Pre-GP Ratio Adani Ports and Special Economic Zone Ltd. - } \\
\text { Post- GP Ratio Adani Ports and Special Economic Zone Ltd. }\end{array}$ & -29.003 & 14.796 & 5.592 & -5.186 & 0.002 & Accepted \\
\hline Pair 3 & $\begin{array}{l}\text { Pre-GP Ratio Ambuja Cements Ltd. - Post- GP Ratio Ambuja } \\
\text { Cements Ltd. }\end{array}$ & -1.870 & 15.043 & 5.686 & -0.329 & 0.753 & Rejected \\
\hline Pair 4 & $\begin{array}{l}\text { Pre-GP Ratio Asian Paints Ltd. - Post- GP Ratio Asian Paints } \\
\text { Ltd. }\end{array}$ & -2.467 & 3.809 & 1.440 & -1.714 & 0.137 & Rejected \\
\hline Pair 5 & Pre-GP Ratio Axis Bank Ltd. - Post- GP Ratio Axis Bank Ltd. & -1.873 & 1.720 & 0.650 & -2.881 & 0.028 & Accepted \\
\hline Pair 6 & Pre-GP Ratio Bajaj Auto Ltd. - Post-GP Ratio Bajaj Auto Ltd. & -5.504 & 1.559 & 0.589 & -9.342 & 0.000 & Accepted \\
\hline Pair 7 & $\begin{array}{l}\text { Pre-GP Ratio Bank Of Baroda - Post- GP Ratio Bank Of } \\
\text { Baroda }\end{array}$ & -1.229 & 0.978 & 0.370 & -3.323 & 0.016 & Accepted \\
\hline Pair 8 & $\begin{array}{l}\text { Pre-GP Ratio Bharat Heavy Electricals Ltd. - Post- GP Ratio } \\
\text { Bharat Heavy Electricals Ltd. }\end{array}$ & -5.237 & 8.061 & 3.047 & -1.719 & 0.136 & Rejected \\
\hline Pair 9 & $\begin{array}{l}\text { Pre-GP Ratio Bharat Petroleum Corpn. Ltd. - Post- GP Ratio } \\
\text { Bharat Petroleum Corpn. Ltd. }\end{array}$ & 1.830 & 2.792 & 1.055 & 1.734 & 0.134 & Rejected \\
\hline Pair 10 & $\begin{array}{l}\text { Pre-GP Ratio BhartiAirtel Ltd. - Post- GP Ratio BhartiAirtel } \\
\text { Ltd. }\end{array}$ & 2.761 & 16.543 & 6.252 & 0.442 & 0.674 & Rejected \\
\hline Pair 11 & Pre-GP Ratio Bosch Ltd. - Post- GP Ratio Bosch Ltd. & 4.954 & 6.656 & 2.516 & 1.969 & 0.096 & Rejected \\
\hline Pair 12 & Pre-GP Ratio Cairn India Ltd. - Post-GP Ratio Cairn India Ltd. & 2435.906 & 2884.542 & 1090.254 & 2.234 & 0.067 & Rejected \\
\hline Pair 13 & Pre-GP Ratio Cipla Ltd. - Post- GP Ratio Cipla Ltd. & -0.159 & 7.131 & 2.695 & -0.059 & 0.955 & Rejected \\
\hline Pair 14 & Pre-GP Ratio Coal India Ltd. - Post- GP Ratio Coal India Ltd. & -107.839 & 50.518 & 19.094 & -5.648 & 0.001 & Accepted \\
\hline Pair 15 & $\begin{array}{l}\text { Pre-GP Ratio Dr. Reddy'S Laboratories Ltd. - Post- GP Ratio } \\
\text { Dr. Reddy'S Laboratories Ltd. }\end{array}$ & -0.860 & 14.939 & 5.646 & -0.152 & 0.884 & Rejected \\
\hline Pair 16 & $\begin{array}{l}\text { Pre-GP Ratio G A I L (India) Ltd. - Post- GP Ratio G A I L } \\
\text { (India) Ltd. }\end{array}$ & 6.883 & 10.697 & 4.043 & 1.702 & 0.140 & Rejected \\
\hline Pair 17 & $\begin{array}{l}\text { Pre-GP Ratio Grasim Industries Ltd. - Post- GP Ratio Grasim } \\
\text { Industries Ltd. }\end{array}$ & -6.870 & 9.294 & 3.513 & -1.956 & 0.098 & Rejected \\
\hline Pair 18 & $\begin{array}{l}\text { Pre-GP Ratio H C L Technologies Ltd. - Post- GP Ratio H C L } \\
\text { Technologies Ltd. }\end{array}$ & 0.304 & 18.204 & 6.881 & 0.044 & 0.966 & Rejected \\
\hline Pair 19 & $\begin{array}{l}\text { Pre-GP Ratio H D F C Bank Ltd. - Post- GP Ratio H D F C } \\
\text { Bank Ltd. }\end{array}$ & -2.224 & 2.823 & 1.067 & -2.084 & 0.082 & Rejected \\
\hline Pair 20 & $\begin{array}{l}\text { Pre-GP Ratio Hero Motocorp Ltd. - Post- GP Ratio Hero } \\
\text { Motocorp Ltd. }\end{array}$ & 2.701 & 5.476 & 2.070 & 1.305 & 0.240 & Rejected \\
\hline Pair 21 & $\begin{array}{l}\text { Pre-GP Ratio Hindalco Industries Ltd. - Post- GP Ratio } \\
\text { Hindalco Industries Ltd. }\end{array}$ & 12.090 & 13.255 & 5.010 & 2.413 & 0.052 & Rejected \\
\hline Pair 22 & $\begin{array}{l}\text { Pre-GP Ratio Hindustan Unilever Ltd. - Post- GP Ratio } \\
\text { Hindustan Unilever Ltd. }\end{array}$ & 1.180 & 6.797 & 2.569 & 0.459 & 0.662 & Rejected \\
\hline Pair 23 & $\begin{array}{l}\text { Pre-GP Ratio Housing Development Finance Corpn. Ltd. - } \\
\text { Post- GP Ratio Housing Development Finance Corpn. Ltd. }\end{array}$ & -59.730 & 20.138 & 7.611 & -7.848 & 0.000 & Accepted \\
\hline Pair 24 & $\begin{array}{l}\text { Pre-GP Ratio I C I C I Bank Ltd. - Post- GP Ratio I C I C I Bank } \\
\text { Ltd. }\end{array}$ & -3.797 & 1.280 & 0.484 & -7.851 & 0.000 & Accepted \\
\hline Pair 25 & Pre-GP Ratio I T C Ltd. - Post- GP Ratio I T C Ltd. & -0.101 & 9.705 & 3.668 & -0.028 & 0.979 & Rejected \\
\hline Pair 26 & $\begin{array}{l}\text { Pre-GP Ratio Idea Cellular Ltd. - Post- GP Ratio Idea Cellular } \\
\text { Ltd. }\end{array}$ & -6.623 & 14.341 & 5.420 & -1.222 & 0.268 & Rejected \\
\hline Pair 27 & $\begin{array}{l}\text { Pre-GP Ratio Indusind Bank Ltd. - Post- GP Ratio Indusind } \\
\text { Bank Ltd. }\end{array}$ & -2.447 & 0.962 & 0.364 & -6.729 & 0.001 & Accepted \\
\hline Pair 29 & $\begin{array}{l}\text { Pre-GP Ratio Kotak Mahindra Bank Ltd. - Post- GP Ratio } \\
\text { Kotak Mahindra Bank Ltd. }\end{array}$ & -3.196 & 1.470 & 0.556 & -5.752 & 0.001 & Accepted \\
\hline Pair 30 & $\begin{array}{l}\text { Pre-GP Ratio Larsen \& Toubro Ltd. - Post- GP Ratio Larsen \& } \\
\text { Toubro Ltd. }\end{array}$ & -2.427 & 3.699 & 1.398 & -1.736 & 0.133 & Rejected \\
\hline
\end{tabular}


ISSN (O) : 2456-6675, ISSN (P) : 0972-8058

\begin{tabular}{|c|c|c|c|c|c|c|c|}
\hline Pair 31 & Pre-GP Ratio Lupin Ltd. - Post- GP Ratio Lupin Ltd. & -8.980 & 5.081 & 1.920 & -4.676 & 0.003 & Accepted \\
\hline Pair 32 & $\begin{array}{l}\text { Pre-GP Ratio Mahindra \& Mahindra Ltd. - Post- GP Ratio } \\
\text { Mahindra \& Mahindra Ltd. }\end{array}$ & -2.580 & 5.069 & 1.916 & -1.347 & 0.227 & Rejected \\
\hline Pair 33 & $\begin{array}{l}\text { Pre-GP Ratio Maruti Suzuki India Ltd. - Post- GP Ratio Maruti } \\
\text { Suzuki India Ltd. }\end{array}$ & 1.647 & 8.891 & 3.361 & 0.490 & 0.641 & Rejected \\
\hline Pair 34 & Pre-GP Ratio N T P C Ltd. - Post- GP Ratio N T P C Ltd. & 6.221 & 15.002 & 5.670 & 1.097 & 0.315 & Rejected \\
\hline Pair 35 & $\begin{array}{l}\text { Pre-GP Ratio Oil \& Natural Gas Corpn. Ltd. - Post- GP Ratio } \\
\text { Oil \& Natural Gas Corpn. Ltd. }\end{array}$ & -1.089 & 16.130 & 6.097 & -0.179 & 0.864 & Rejected \\
\hline Pair 36 & $\begin{array}{l}\text { Pre-GP Ratio Power Grid Corpn. Of India Ltd. - Post- GP } \\
\text { Ratio Power Grid Corpn. Of India Ltd. }\end{array}$ & 86.120 & 270.571 & 102.266 & 0.842 & 0.432 & Rejected \\
\hline Pair 37 & $\begin{array}{l}\text { Pre-GP Ratio Punjab National Bank - Post- GP Ratio Punjab } \\
\text { National Bank }\end{array}$ & -2.053 & 1.247 & 0.471 & -4.355 & 0.005 & Accepted \\
\hline Pair 38 & $\begin{array}{l}\text { Pre-GP Ratio Reliance Industries Ltd. - Post- GP Ratio } \\
\text { Reliance Industries Ltd. }\end{array}$ & 2.837 & 6.741 & 2.548 & 1.114 & 0.308 & Rejected \\
\hline Pair 39 & $\begin{array}{l}\text { Pre-GP Ratio State Bank Of India - Post- GP Ratio State Bank } \\
\text { Of India }\end{array}$ & -0.926 & 1.843 & 0.697 & -1.329 & 0.232 & Rejected \\
\hline Pair 40 & $\begin{array}{l}\text { Pre-GP Ratio Sun Pharmaceutical Inds. Ltd. - Post- GP Ratio } \\
\text { Sun Pharmaceutical Inds. Ltd. }\end{array}$ & 5.164 & 16.437 & 6.212 & 0.831 & 0.438 & Rejected \\
\hline Pair 41 & $\begin{array}{l}\text { Pre-GP Ratio Tata Consultancy Services Ltd. - Post- GP Ratio } \\
\text { Tata Consultancy Services Ltd. }\end{array}$ & -1.440 & 7.625 & 2.882 & -0.500 & 0.635 & Rejected \\
\hline Pair 42 & $\begin{array}{l}\text { Pre-GP Ratio Tata Motors Ltd. - Post- GP Ratio Tata Motors } \\
\text { Ltd. }\end{array}$ & 2.467 & 8.488 & 3.208 & 0.769 & 0.471 & Rejected \\
\hline Pair 43 & $\begin{array}{l}\text { Pre-GP Ratio Tata Power Co. Ltd. - Post- GP Ratio Tata Power } \\
\text { Co. Ltd. }\end{array}$ & 2.700 & 6.054 & 2.288 & 1.180 & 0.283 & Rejected \\
\hline Pair 44 & Pre-GP Ratio Tata Steel Ltd. - Post- GP Ratio Tata Steel Ltd. & -8.346 & 17.858 & 6.750 & -1.236 & 0.262 & Rejected \\
\hline Pair 45 & $\begin{array}{l}\text { Pre-GP Ratio Tech Mahindra Ltd. - Post- GP Ratio Tech } \\
\text { Mahindra Ltd. }\end{array}$ & -5.187 & 8.356 & 3.158 & -1.642 & 0.152 & Rejected \\
\hline Pair 46 & $\begin{array}{l}\text { Pre-GP Ratio Ultratech Cement Ltd. - Post- GP Ratio Ultratech } \\
\text { Cement Ltd. }\end{array}$ & -10.483 & 9.103 & 3.440 & -3.047 & 0.023 & Accepted \\
\hline Pair 47 & Pre-GP Ratio Vedanta Ltd. - Post- GP Ratio Vedanta Ltd. & -11.583 & 45.827 & 17.321 & -0.669 & 0.529 & Rejected \\
\hline Pair 48 & Pre-GP Ratio Wipro Ltd. - Post- GP Ratio Wipro Ltd. & 2.387 & 7.218 & 2.728 & 0.875 & 0.415 & Rejected \\
\hline Pair 49 & Pre-GP Ratio Yes Bank Ltd. - Post- GP Ratio Yes Bank Ltd. & -4.346 & 3.036 & 1.147 & -3.787 & 0.009 & Accepted \\
\hline Pair 50 & $\begin{array}{l}\text { Pre-GP Ratio Zee Entertainment Enterprises Ltd. - Post- GP } \\
\text { Ratio Zee Entertainment Enterprises Ltd. }\end{array}$ & -7.440 & 12.375 & 4.677 & -1.591 & 0.163 & Rejected \\
\hline
\end{tabular}

The results of Paired Samples t-Test in Table-2 depict that the alternative hypotheses of 36 companies have been rejected while it has been accepted for 14 companies. Hence statistically there is no significance impact of implementation of clause- 49 of corporate governance on the gross profit ratio of majority of nifty-50 companies in India. The industries were further clustered on the basis of acceptance and rejection of the hypotheses. It has been observed that gross profit ratios of the firms affected significantly are banks.

H3 : There is significance difference in the net profit ratio of Nifty-50 companies between pre and post implementation of clause- 49 of corporate governance in India. 
ISSN (O) : 2456-6675, ISSN (P) : 0972-8058

\section{Table-3 Paired Samples Test}

\begin{tabular}{|c|c|c|c|c|c|c|c|}
\hline & \multicolumn{3}{|c|}{ Paired Differences } & \multirow{2}{*}{$\begin{array}{c}\mathrm{t}- \\
\text { value }\end{array}$} & \multirow{2}{*}{$\begin{array}{l}\text { Sig. } \\
(2- \\
\text { tailed) }\end{array}$} & \multirow{2}{*}{$\begin{array}{c}\text { Result of } \\
\text { Hypothese } \\
\text { s }\end{array}$} \\
\hline & & Mean & $\begin{array}{c}\text { Std. } \\
\text { Deviation }\end{array}$ & $\begin{array}{l}\text { Std. Error } \\
\text { Mean }\end{array}$ & & & \\
\hline Pair 1 & Pre-NP Ratio A C C Ltd. - Post- NP Ratio A C C Ltd. & -10.969 & 10.660 & 3.769 & -2.910 & 0.023 & Accepted \\
\hline Pair 2 & $\begin{array}{l}\text { Pre-NP Ratio Adani Ports and Special Economic Zone Ltd. - Post- } \\
\text { NP Ratio Adani Ports and Special Economic Zone Ltd. }\end{array}$ & -37.813 & 11.080 & 3.917 & -9.653 & 0.000 & Accepted \\
\hline Pair 3 & $\begin{array}{l}\text { Pre-NP Ratio Ambuja Cements Ltd. - Post- NP Ratio Ambuja } \\
\text { Cements Ltd. }\end{array}$ & -0.426 & 8.465 & 2.993 & -0.142 & 0.891 & Rejected \\
\hline Pair 4 & Pre-NP Ratio Asian Paints Ltd. - Post- NP Ratio Asian Paints Ltd. & -3.245 & 1.734 & 0.613 & -5.293 & 0.001 & Accepted \\
\hline Pair 5 & Pre-NP Ratio Axis Bank Ltd. - Post- NP Ratio Axis Bank Ltd. & -6.724 & 2.371 & 0.838 & -8.022 & 0.000 & Accepted \\
\hline Pair 6 & Pre-NP Ratio Bajaj Auto Ltd. - Post-NP Ratio Bajaj Auto Ltd. & -5.906 & 3.971 & 1.404 & -4.207 & 0.004 & Accepted \\
\hline Pair 7 & Pre-NP Ratio Bank Of Baroda - Post- NP Ratio Bank Of Baroda & -6.226 & 3.030 & 1.071 & -5.813 & 0.001 & Accepted \\
\hline Pair 8 & $\begin{array}{l}\text { Pre-NP Ratio Bharat Heavy Electricals Ltd. - Post- NP Ratio } \\
\text { Bharat Heavy Electricals Ltd. }\end{array}$ & -6.013 & 4.317 & 1.526 & -3.939 & 0.006 & Accepted \\
\hline Pair 9 & $\begin{array}{l}\text { Pre-NP Ratio Bharat Petroleum Corpn. Ltd. - Post- NP Ratio } \\
\text { Bharat Petroleum Corpn. Ltd. }\end{array}$ & 0.799 & 1.354 & 0.479 & 1.668 & 0.139 & Rejected \\
\hline Pair 10 & Pre-NP Ratio BhartiAirtel Ltd. - Post- NP Ratio BhartiAirtel Ltd. & -14.813 & 12.250 & 4.331 & -3.420 & 0.011 & Accepted \\
\hline Pair 11 & Pre-NP Ratio Bosch Ltd. - Post- NP Ratio Bosch Ltd. & -4.349 & 5.460 & 1.930 & -2.253 & 0.059 & Rejected \\
\hline Pair 12 & Pre-NP Ratio Cairn India Ltd. - Post-NP Ratio Cairn India Ltd. & 1113.215 & 3152.698 & 1114.647 & 0.999 & 0.351 & Rejected \\
\hline Pair 13 & Pre-NP Ratio Cipla Ltd. - Post- NP Ratio Cipla Ltd. & 0.043 & 3.644 & 1.288 & 0.033 & 0.975 & Rejected \\
\hline Pair 14 & Pre-NP Ratio Coal India Ltd. - Post- NP Ratio Coal India Ltd. & -1187.510 & 1194.632 & 422.366 & -2.812 & 0.026 & Accepted \\
\hline Pair 15 & $\begin{array}{l}\text { Pre-NP Ratio Dr. Reddy'S Laboratories Ltd. - Post- NP Ratio Dr. } \\
\text { Reddy'S Laboratories Ltd. }\end{array}$ & -1.346 & 10.203 & 3.607 & -0.373 & 0.720 & Rejected \\
\hline Pair 16 & $\begin{array}{l}\text { Pre-NP Ratio G A I L (India) Ltd. - Post- NP Ratio G A I L (India) } \\
\text { Ltd. }\end{array}$ & 1.419 & 4.695 & 1.660 & 0.855 & 0.421 & Rejected \\
\hline Pair 17 & $\begin{array}{l}\text { Pre-NP Ratio Grasim Industries Ltd. - Post- NP Ratio Grasim } \\
\text { Industries Ltd. }\end{array}$ & -10.710 & 4.654 & 1.645 & -6.509 & 0.000 & Accepted \\
\hline Pair 18 & $\begin{array}{l}\text { Pre-NP Ratio H C L Technologies Ltd. - Post- NP Ratio H C L } \\
\text { Technologies Ltd. }\end{array}$ & 14.225 & 19.177 & 6.780 & 2.098 & 0.074 & Rejected \\
\hline Pair 19 & $\begin{array}{l}\text { Pre-NP Ratio H D F C Bank Ltd. - Post- NP Ratio H D F C Bank } \\
\text { Ltd. }\end{array}$ & -1.166 & 2.631 & 0.930 & -1.254 & 0.250 & Rejected \\
\hline Pair 20 & $\begin{array}{l}\text { Pre-NP Ratio Hero Motocorp Ltd. - Post- NP Ratio Hero Motocorp } \\
\text { Ltd. }\end{array}$ & -0.320 & 2.662 & 0.941 & -0.340 & 0.744 & Rejected \\
\hline Pair 21 & $\begin{array}{l}\text { Pre-NP Ratio Hindalco Industries Ltd. - Post- NP Ratio Hindalco } \\
\text { Industries Ltd. }\end{array}$ & 10.155 & 5.863 & 2.073 & 4.899 & 0.002 & Accepted \\
\hline Pair 22 & $\begin{array}{l}\text { Pre-NP Ratio Hindustan Unilever Ltd. - Post- NP Ratio Hindustan } \\
\text { Unilever Ltd. }\end{array}$ & 0.321 & 3.806 & 1.345 & 0.239 & 0.818 & Rejected \\
\hline Pair 23 & $\begin{array}{l}\text { Pre-NP Ratio Housing Development Finance Corpn. Ltd. - Post- } \\
\text { NP Ratio Housing Development Finance Corpn. Ltd. }\end{array}$ & -1.565 & 7.364 & 2.603 & -0.601 & 0.567 & Rejected \\
\hline Pair 24 & $\begin{array}{l}\text { Pre-NP Ratio I C I C I Bank Ltd. - Post- NP Ratio I C I C I Bank } \\
\text { Ltd. }\end{array}$ & -3.604 & 3.908 & 1.382 & -2.608 & 0.035 & Accepted \\
\hline Pair 25 & Pre-NP Ratio I T C Ltd. - Post- NP Ratio I T C Ltd. & 0.768 & 2.308 & 0.816 & 0.941 & 0.378 & Rejected \\
\hline Pair 26 & Pre-NP Ratio Idea Cellular Ltd. - Post- NP Ratio Idea Cellular Ltd. & -16.805 & 14.294 & 5.054 & -3.325 & 0.013 & Accepted \\
\hline Pair 27 & $\begin{array}{l}\text { Pre-NP Ratio Indusind Bank Ltd. - Post- NP Ratio Indusind Bank } \\
\text { Ltd. }\end{array}$ & -2.330 & 6.226 & 2.201 & -1.058 & 0.325 & Rejected \\
\hline Pair 28 & Pre-NP Ratio Infosys Ltd. - Post- NP Ratio Infosys Ltd. & 2.909 & 2.068 & 0.731 & 3.978 & 0.005 & Accepted \\
\hline Pair 29 & $\begin{array}{l}\text { Pre-NP Ratio Kotak Mahindra Bank Ltd. - Post- NP Ratio Kotak } \\
\text { Mahindra Bank Ltd. }\end{array}$ & 9.658 & 11.434 & 4.043 & 2.389 & 0.048 & Accepted \\
\hline Pair 30 & $\begin{array}{l}\text { Pre-NP Ratio Larsen \& Toubro Ltd. - Post- NP Ratio Larsen \& } \\
\text { Toubro Ltd. }\end{array}$ & -3.794 & 2.298 & 0.812 & -4.670 & 0.002 & Accepted \\
\hline Pair 31 & Pre-NP Ratio Lupin Ltd. - Post- NP Ratio Lupin Ltd. & -9.194 & 3.551 & 1.255 & -7.324 & 0.000 & Accepted \\
\hline
\end{tabular}




\begin{tabular}{|c|c|c|c|c|c|c|c|}
\hline Pair 32 & $\begin{array}{l}\text { Pre-NP Ratio Mahindra \& Mahindra Ltd. - Post- NP Ratio } \\
\text { Mahindra \& Mahindra Ltd. }\end{array}$ & -4.041 & 3.363 & 1.189 & -3.399 & 0.011 & Accepted \\
\hline Pair 33 & $\begin{array}{l}\text { Pre-NP Ratio Maruti Suzuki India Ltd. - Post- NP Ratio Maruti } \\
\text { Suzuki India Ltd. }\end{array}$ & -4.295 & 5.675 & 2.006 & -2.141 & 0.070 & Rejected \\
\hline Pair 34 & Pre-NP Ratio N T P C Ltd. - Post- NP Ratio N T P C Ltd. & 3.009 & 4.674 & 1.653 & 1.821 & 0.111 & Rejected \\
\hline Pair 35 & $\begin{array}{l}\text { Pre-NP Ratio Oil \& Natural Gas Corpn. Ltd. - Post- NP Ratio Oil } \\
\text { \& Natural Gas Corpn. Ltd. }\end{array}$ & -1.089 & 3.395 & 1.200 & -0.907 & 0.395 & Rejected \\
\hline Pair 36 & $\begin{array}{l}\text { Pre-NP Ratio Power Grid Corpn. Of India Ltd. - Post- NP Ratio } \\
\text { Power Grid Corpn. Of India Ltd. }\end{array}$ & 137.326 & 389.149 & 137.585 & 0.998 & 0.351 & Rejected \\
\hline Pair 37 & $\begin{array}{l}\text { Pre-NP Ratio Punjab National Bank - Post- NP Ratio Punjab } \\
\text { National Bank }\end{array}$ & -3.401 & 5.433 & 1.921 & -1.771 & 0.120 & Rejected \\
\hline Pair 38 & $\begin{array}{l}\text { Pre-NP Ratio Reliance Industries Ltd. - Post- NP Ratio Reliance } \\
\text { Industries Ltd. }\end{array}$ & 1.070 & 3.663 & 1.295 & 0.826 & 0.436 & Rejected \\
\hline Pair 39 & $\begin{array}{l}\text { Pre-NP Ratio State Bank Of India - Post- NP Ratio State Bank Of } \\
\text { India }\end{array}$ & -2.439 & 3.377 & 1.194 & -2.042 & 0.080 & Rejected \\
\hline Pair 40 & $\begin{array}{l}\text { Pre-NP Ratio Sun Pharmaceutical Inds. Ltd. - Post- NP Ratio Sun } \\
\text { Pharmaceutical Inds. Ltd. }\end{array}$ & 4.305 & 54.066 & 19.115 & 0.225 & 0.828 & Rejected \\
\hline Pair 41 & $\begin{array}{l}\text { Pre-NP Ratio Tata Consultancy Services Ltd. - Post- NP Ratio Tata } \\
\text { Consultancy Services Ltd. }\end{array}$ & -0.820 & 4.725 & 1.670 & -0.491 & 0.639 & Rejected \\
\hline Pair 42 & Pre-NP Ratio Tata Motors Ltd. - Post- NP Ratio Tata Motors Ltd. & -2.025 & 7.091 & 2.507 & -0.808 & 0.446 & Rejected \\
\hline Pair 43 & $\begin{array}{l}\text { Pre-NP Ratio Tata Power Co. Ltd. - Post- NP Ratio Tata Power Co. } \\
\text { Ltd. }\end{array}$ & -0.970 & 2.581 & 0.913 & -1.063 & 0.323 & Rejected \\
\hline Pair 44 & Pre-NP Ratio Tata Steel Ltd. - Post- NP Ratio Tata Steel Ltd. & -7.455 & 11.038 & 3.902 & -1.910 & 0.098 & Rejected \\
\hline Pair 45 & $\begin{array}{l}\text { Pre-NP Ratio Tech Mahindra Ltd. - Post- NP Ratio Tech Mahindra } \\
\text { Ltd. }\end{array}$ & 7.948 & 8.105 & 2.866 & 2.773 & 0.028 & Accepted \\
\hline Pair 46 & $\begin{array}{l}\text { Pre-NP Ratio Ultratech Cement Ltd. - Post- NP Ratio Ultratech } \\
\text { Cement Ltd. }\end{array}$ & -12.931 & 4.459 & 1.577 & -8.202 & 0.000 & Accepted \\
\hline Pair 47 & Pre-NP Ratio Vedanta Ltd. - Post- NP Ratio Vedanta Ltd. & -15.618 & 27.087 & 9.577 & -1.631 & 0.147 & Rejected \\
\hline Pair 48 & Pre-NP Ratio Wipro Ltd. - Post- NP Ratio Wipro Ltd. & 3.070 & 2.618 & 0.926 & 3.317 & 0.013 & Accepted \\
\hline Pair 49 & Pre-NP Ratio Yes Bank Ltd. - Post- NP Ratio Yes Bank Ltd. & -13.410 & 7.624 & 2.695 & -4.975 & 0.002 & Accepted \\
\hline Pair 50 & $\begin{array}{l}\text { Pre-NP Ratio Zee Entertainment Enterprises Ltd. - Post- NP Ratio } \\
\text { Zee Entertainment Enterprises Ltd. }\end{array}$ & -3.311 & 11.283 & 3.989 & -0.830 & 0.434 & Rejected \\
\hline
\end{tabular}

The results of Paired Samples t-Test in Table- 3 depict that the alternative hypotheses of 28 companies have been rejected while it has been accepted for 22 companies. Hence statistically there is no significance impact of implementation of clause- 49 of corporate governance on the net profit ratio of majority of nifty-50 companies in India. The industries were further clustered on the basis of acceptance and rejection of the hypotheses. It has been observed that net profit ratios of the firms affected significantly are not from a particulate industry.

H4 : There is significance difference in the financial leverage of Nifty-50 companies between pre and post implementation of clause- 49 of corporate governance in India. 


\begin{tabular}{|c|c|c|c|c|c|c|c|}
\hline \multicolumn{8}{|c|}{ Table-4 Paired Samples Test } \\
\hline & & \multicolumn{3}{|c|}{ Paired Differences } & \multirow{2}{*}{$\begin{array}{c}\mathrm{t}- \\
\text { value }\end{array}$} & \multirow{2}{*}{$\begin{array}{c}\text { Sig. } \\
\text { (2-tailed) }\end{array}$} & \multirow[b]{2}{*}{$\begin{array}{c}\text { Result of } \\
\text { Hypotheses }\end{array}$} \\
\hline & & Mean & $\begin{array}{c}\text { Std. } \\
\text { Deviation }\end{array}$ & $\begin{array}{l}\text { Std. Error } \\
\text { Mean }\end{array}$ & & & \\
\hline Pair 1 & Pre-FL A C C Ltd. - Post- FL A C C Ltd. & 1.204 & 3.222 & 1.139 & 1.057 & 0.326 & Rejected \\
\hline Pair 2 & $\begin{array}{l}\text { Pre-FL Adani Ports and Special Economic Zone Ltd. - Post- } \\
\text { FL Adani Ports and Special Economic Zone Ltd. }\end{array}$ & -0.753 & 0.794 & 0.281 & -2.679 & 0.032 & Accepted \\
\hline Pair 3 & $\begin{array}{l}\text { Pre-FL Ambuja Cements Ltd. - Post- FL Ambuja Cements } \\
\text { Ltd. }\end{array}$ & 0.691 & 0.555 & 0.196 & 3.521 & 0.010 & Accepted \\
\hline Pair 4 & Pre-FL Asian Paints Ltd. - Post- FL Asian Paints Ltd. & 0.204 & 0.067 & 0.024 & 8.634 & 0.000 & Accepted \\
\hline Pair 5 & Pre-FL Axis Bank Ltd. - Post- FL Axis Bank Ltd. & 2.365 & 1.802 & 0.637 & 3.712 & 0.008 & Accepted \\
\hline Pair 6 & Pre-FL Bajaj Auto Ltd. - Post-FL Bajaj Auto Ltd. & -0.445 & 0.252 & 0.089 & -5.000 & 0.002 & Accepted \\
\hline Pair 7 & Pre-FL Bank Of Baroda - Post- FL Bank Of Baroda & 1.789 & 2.527 & 0.893 & 2.002 & 0.085 & Rejected \\
\hline Pair 8 & $\begin{array}{l}\text { Pre-FL Bharat Heavy Electricals Ltd. - Post- FL Bharat Heavy } \\
\text { Electricals Ltd. }\end{array}$ & 0.291 & 0.477 & 0.169 & 1.726 & 0.128 & Rejected \\
\hline Pair 9 & $\begin{array}{l}\text { Pre-FL Bharat Petroleum Corpn. Ltd. - Post- FL Bharat } \\
\text { Petroleum Corpn. Ltd. }\end{array}$ & -0.906 & 1.797 & 0.635 & -1.426 & 0.197 & Rejected \\
\hline Pair 10 & Pre-FL BhartiAirtel Ltd. - Post- FL BhartiAirtel Ltd. & 42.156 & 49.064 & 17.347 & 2.430 & 0.045 & Accepted \\
\hline Pair 11 & Pre-FL Bosch Ltd. - Post- FL Bosch Ltd. & 0.438 & 0.369 & 0.131 & 3.352 & 0.012 & Accepted \\
\hline Pair 12 & Pre-FL Cairn India Ltd. - Post-FL Cairn India Ltd. & -3.028 & 7.098 & 2.510 & -1.206 & 0.267 & Rejected \\
\hline Pair 13 & Pre-FL Cipla Ltd. - Post- FL Cipla Ltd. & -0.138 & 0.126 & 0.045 & -3.081 & 0.018 & Accepted \\
\hline Pair 14 & Pre-FL Coal India Ltd. - Post- FL Coal India Ltd. & 0.799 & 0.661 & 0.234 & 3.418 & 0.011 & Accepted \\
\hline Pair 15 & $\begin{array}{l}\text { Pre-FL Dr. Reddy'S Laboratories Ltd. - Post- FL Dr. Reddy'S } \\
\text { Laboratories Ltd. }\end{array}$ & 0.711 & 1.548 & 0.547 & 1.300 & 0.235 & Rejected \\
\hline Pair 16 & Pre-FL G A I L (India) Ltd. - Post- FL G A I L (India) Ltd. & 0.141 & 0.166 & 0.059 & 2.401 & 0.047 & Accepted \\
\hline Pair 17 & $\begin{array}{l}\text { Pre-FL Grasim Industries Ltd. - Post- FL Grasim Industries } \\
\text { Ltd. }\end{array}$ & 1.178 & 1.277 & 0.452 & 2.607 & 0.035 & Accepted \\
\hline Pair 18 & $\begin{array}{l}\text { Pre-FL H C L Technologies Ltd. - Post- FL H C L } \\
\text { Technologies Ltd. }\end{array}$ & -0.080 & 0.237 & 0.084 & -0.956 & 0.371 & Rejected \\
\hline Pair 19 & Pre-FL H D F C Bank Ltd. - Post- FL H D F C Bank Ltd. & -0.158 & 0.532 & 0.188 & -0.837 & 0.430 & Rejected \\
\hline Pair 20 & Pre-FL Hero Motocorp Ltd. - Post- FL Hero Motocorp Ltd. & -0.108 & 0.192 & 0.068 & -1.580 & 0.158 & Rejected \\
\hline Pair 21 & $\begin{array}{l}\text { Pre-FL Hindalco Industries Ltd. - Post- FL Hindalco } \\
\text { Industries Ltd. }\end{array}$ & -0.115 & 0.232 & 0.082 & -1.400 & 0.204 & Rejected \\
\hline Pair 22 & $\begin{array}{l}\text { Pre-FL Hindustan Unilever Ltd. - Post- FL Hindustan } \\
\text { Unilever Ltd. }\end{array}$ & 0.034 & 0.057 & 0.020 & 1.688 & 0.135 & Rejected \\
\hline Pair 23 & $\begin{array}{l}\text { Pre-FL Housing Development Finance Corpn. Ltd. - Post- FL } \\
\text { Housing Development Finance Corpn. Ltd. }\end{array}$ & 0.551 & 0.860 & 0.304 & 1.813 & 0.113 & Rejected \\
\hline Pair 24 & Pre-FL I C I C I Bank Ltd. - Post- FL I C I C I Bank Ltd. & 1.853 & 3.543 & 1.253 & 1.479 & 0.183 & Rejected \\
\hline Pair 25 & Pre-FL I T C Ltd. - Post- FL I T C Ltd. & 0.075 & 0.079 & 0.028 & 2.688 & 0.031 & Accepted \\
\hline Pair 26 & Pre-FL Idea Cellular Ltd. - Post- FL Idea Cellular Ltd. & -0.490 & 8.780 & 3.104 & -0.158 & 0.879 & Rejected \\
\hline Pair 27 & Pre-FL Indusind Bank Ltd. - Post- FL Indusind Bank Ltd. & 3.584 & 6.052 & 2.140 & 1.675 & 0.138 & Rejected \\
\hline Pair 28 & Pre-FL Infosys Ltd. - Post- FL Infosys Ltd. & 0.081 & 0.027 & 0.010 & 8.524 & 0.000 & Accepted \\
\hline Pair 29 & $\begin{array}{l}\text { Pre-FL Kotak Mahindra Bank Ltd. - Post- FL Kotak Mahindra } \\
\text { Bank Ltd. }\end{array}$ & -1.068 & 1.454 & 0.514 & -2.077 & 0.076 & Rejected \\
\hline Pair 30 & Pre-FL Larsen \& Toubro Ltd. - Post- FL Larsen \& Toubro Ltd. & 0.955 & 0.877 & 0.310 & 3.080 & 0.018 & Accepted \\
\hline
\end{tabular}




\begin{tabular}{|c|c|c|c|c|c|c|c|}
\hline Pair 31 & Pre-FL Lupin Ltd. - Post- FL Lupin Ltd. & 0.853 & 0.406 & 0.144 & 5.939 & 0.001 & Accepted \\
\hline Pair 32 & $\begin{array}{l}\text { Pre-FL Mahindra \& Mahindra Ltd. - Post- FL Mahindra \& } \\
\text { Mahindra Ltd. }\end{array}$ & 1.034 & 0.962 & 0.340 & 3.038 & 0.019 & Accepted \\
\hline Pair 33 & $\begin{array}{l}\text { Pre-FL Maruti Suzuki India Ltd. - Post- FL Maruti Suzuki } \\
\text { India Ltd. }\end{array}$ & 0.471 & 1.988 & 0.703 & 0.671 & 0.524 & Rejected \\
\hline Pair 34 & Pre-FL N T P C Ltd. - Post- FL N T P C Ltd. & 0.296 & 0.302 & 0.107 & 2.776 & 0.027 & Accepted \\
\hline Pair 35 & $\begin{array}{l}\text { Pre-FL Oil \& Natural Gas Corpn. Ltd. - Post- FL Oil \& } \\
\text { Natural Gas Corpn. Ltd. }\end{array}$ & -0.078 & 0.515 & 0.182 & -0.426 & 0.683 & Rejected \\
\hline Pair 36 & $\begin{array}{l}\text { Pre-FL Power Grid Corpn. Of India Ltd. - Post- FL Power } \\
\text { Grid Corpn. Of India Ltd. }\end{array}$ & 0.410 & 0.372 & 0.132 & 3.117 & 0.017 & Accepted \\
\hline Pair 37 & $\begin{array}{l}\text { Pre-FL Punjab National Bank - Post- FL Punjab National } \\
\text { Bank }\end{array}$ & 1.270 & 3.424 & 1.210 & 1.049 & 0.329 & Rejected \\
\hline Pair 38 & $\begin{array}{l}\text { Pre-FL Reliance Industries Ltd. - Post- FL Reliance Industries } \\
\text { Ltd. }\end{array}$ & 0.346 & 0.246 & 0.087 & 3.983 & 0.005 & Accepted \\
\hline Pair 39 & Pre-FL State Bank Of India - Post- FL State Bank Of India & 1.723 & 2.871 & 1.015 & 1.697 & 0.134 & Rejected \\
\hline Pair 40 & $\begin{array}{l}\text { Pre-FL Sun Pharmaceutical Inds. Ltd. - Post- FL Sun } \\
\text { Pharmaceutical Inds. Ltd. }\end{array}$ & 0.124 & 0.156 & 0.055 & 2.238 & 0.060 & Rejected \\
\hline Pair 41 & $\begin{array}{l}\text { Pre-FL Tata Consultancy Services Ltd. - Post- FL Tata } \\
\text { Consultancy Services Ltd. }\end{array}$ & -0.058 & 0.088 & 0.031 & -1.839 & 0.109 & Rejected \\
\hline Pair 42 & Pre-FL Tata Motors Ltd. - Post- FL Tata Motors Ltd. & -2.935 & 12.293 & 4.346 & -0.675 & 0.521 & Rejected \\
\hline Pair 43 & Pre-FL Tata Power Co. Ltd. - Post- FL Tata Power Co. Ltd. & 0.189 & 0.312 & 0.110 & 1.712 & 0.131 & Rejected \\
\hline Pair 44 & Pre-FL Tata Steel Ltd. - Post- FL Tata Steel Ltd. & 1.031 & 1.369 & 0.484 & 2.131 & 0.071 & Rejected \\
\hline Pair 45 & Pre-FL Tech Mahindra Ltd. - Post- FL Tech Mahindra Ltd. & -0.106 & 0.269 & 0.095 & -1.116 & 0.301 & Rejected \\
\hline Pair 46 & $\begin{array}{l}\text { Pre-FL Ultratech Cement Ltd. - Post- FL Ultratech Cement } \\
\text { Ltd. }\end{array}$ & -0.798 & 4.918 & 1.739 & -0.459 & 0.660 & Rejected \\
\hline Pair 47 & Pre-FL Vedanta Ltd. - Post- FL Vedanta Ltd. & -1.880 & 6.206 & 2.194 & -0.857 & 0.420 & Rejected \\
\hline Pair 48 & Pre-FL Wipro Ltd. - Post- FL Wipro Ltd. & 0.143 & 0.404 & 0.143 & 0.998 & 0.352 & Rejected \\
\hline Pair 49 & Pre-FL Yes Bank Ltd. - Post- FL Yes Bank Ltd. & -1.485 & 1.900 & 0.672 & -2.211 & 0.063 & Rejected \\
\hline Pair 50 & $\begin{array}{l}\text { Pre-FL Zee Entertainment Enterprises Ltd. - Post- FL Zee } \\
\text { Entertainment Enterprises Ltd. }\end{array}$ & 0.249 & 0.311 & 0.110 & 2.261 & 0.058 & Rejected \\
\hline
\end{tabular}

The results of Paired Samples t-Test in Table- 4 depict that the alternative hypotheses of 31 companies have been rejected while it has been accepted for 19 companies. Hence statistically there is no significance impact of implementation of clause-49 of corporate governance on the financial leverage of most of the nifty-50 companies in India. The industries were further clustered on the basis of acceptance and rejection of the hypotheses. It has been observed that financial leverage of the firms affected significantly are not from a particular group of companies.

\section{Conclusion, Limitations and Future Scope}

As per this study the corporate governance (clause49) plays a significant role for the improvement in earnings per share for most of the nifty-50 firms but not protest its impact on other selected parameter for firm performance i.e. gross profit ratio, net profit ratio and financial leverage.

Researchers have taken the current list of Nifty 50 companies, which may not be the same for the entire 16 years of study as some companies keep on changing in the list from time to time. So, data of the companies mentioned in the current list of Nifty 50 index is taken for this research purpose to maintain the consistency, thereby some variation may occurred in the data collected from deferent sources. Other variables were assumed as constant while testing the impact of clause-49 implementation on the performance of the listed companies in India.

A comprehensive research may be done by taking top 100 or top 200 listed companies to find out this 
impact with Individual Industry wise study and/or comparative study may also done to find out the implementation status and its impact on firm performance. A detailed study may also be conducted to list out the important factors in Corporate Governance and their individual and /or cumulative impact on firm performance.

\section{REFERENCES}

1) Anderson, R. C., Mansi, S. A., \& Reeb, D. M. (2004), "Board characteristics, accounting report integrity and the cost of debt", Journal of Accounting and Economics, 37(3), pp.315-342.

2) Al-Mudhaki. J. and Joshi, P. L. (2004), "The Role and Functions of Audit Committees in the Indian Corporate Governance: Empirical Findings", International Journal of Auditing, 8, pp.33-47

3) Akinsulire, O. (2006): Financial Management, Lagos, El-Toda Ventures.

4) Adenikinju, $\mathrm{O}$ and F, Ayorinde (2001): "Ownership structure, corporate governance and corporate performance: The case of Nigerian quoted companies", Unpublished final report presented at the AERC biannual research workshop, Nairobi, Kenya, May.

5) Al- Faki, M (2006): “Transparency and corporate governance for capital market development in Africa: The Nigerian case study", Securities Market Journal, pp.9- 28.

6) Bebchuk, L.A. \& Roe, M.J. (1999), “A theory of path dependence in corporate ownership and governance", Stanford Law Review, 52(1), pp.127170.

7) Bhagat, S and B, Black (2002), "The noncorrelation between board independence and long term firm performance", Journal of Corporation Law, Vol. 27, pp 231- 274.

8) Bokpin, G.A, A, Kyereboah- Coleman and A.Q.Q, Aboagye (2006), “Corporate governance and shareholder wealth maximization: Evidence from listed companies in Ghana", Unpublished paper presented at the 3rd African Finance Journal Conference, Accra, Ghana, 12th - 13th
July.

9) Brown, L.D, J.M, Robinson and M.C. Caylor (2004), "Corporate governance and firm performance", http://www.issproxy.com/pdf/corporate governance.

10) Core, J. E., R. W. Holthausen, and D. F. Larcker. (1999), "Corporate governance, chief executive officer compensation, and firm performance". Journal of Financial Economics, 51 (3), pp. 371-406.

11) Chakrabarti, R., Megginson, W. and Yadav, P. K. (2008) , "Corporate Governance in India". Journal of Applied Corporate Finance, 20, pp. 59-72.

12) Dalton, Dan R., Catherine M. Daily, Alan E. Ellstrand, and Jonathan L. Johnson., (1998).

13) "Metal-Analytic Reviews of Board Composition, Leadership Structure and Financial Performance", http://asp.thecorporatelibrary.net/patterson/Li nkDetail.asp?CatalogID=14289>

14) Dharwardkar, R, George, G and Brandes, P (2000): "Privatization in Emerging Economies: An Agency Perspective", Academy of Management Review, 25(3), 650-669.

15) Dwivedi, N. and Jain, A.K. (2005), “Corporate Governance and Performance of Indian Firms: The Effect of Board Size and Ownership. Employee Responsibilities and Rights Journal", Vol.17, No.3, Sept, pp: 161-172.

16) Elankumaran, S., R. Seal and A. Hashmi (2005), "Transcending Transformation: Enlightening Endeavours at Tata Steel", Journal of Business Ethics, 59, pp. 109-119.

17) Fosberg, R (1989), “Outside directors and managerial monitoring", Akron Business and Economic Review", Vol. 20, pp.24- 32.

18) Freeman, R.E. (1984), "Stakeholder Management: A Strategic Approach", Pitman, Boston, MA.

19) Froud, J., Haslam, C., Johal, S. and Williams, K. (2000), "Shareholder value and the political economy of late capitalism", Economy \& Society, Vol. 29 No. 1, pp. 1-12

20) Ghosh S (2006), “Do board characteristics affect corporate performance? Firm-level evidence for India", Applied Economics Letters, Volume 13, 
Number 7, pp. 435-443(9)

21) Hair, J. E., Anderson, R. E., Tatham, R. L., \& Black W. C. (1998), Multivariate data analysis. (5th ed.). Upper Saddle River, NJ: Prentice-Hall.

22) Jensen, M.C., and W.H. Meckling (1976), "Theory of the Firm: Managerial Behavior, Agency Costs and Ownership Structure", Journal of Financial Economics, October, V.3, 4, pp. 305-360.

23) Kathuria, V. \& Dash S.(1999), "Board Size and Corporate Financial Performance; An Investigation", Vikalpa, Vol 24, No 3, pp.11-17

24) Kimber D. \& Lipton P. (2005). “Corporate governance and business ethics in the AsiaPacific region". Business \& Society, 44(2): 178-210.

25) Khanna, Tarun and Palepu, Krishna (2004), "Globalization and Convergence in Corporate Governance: Evidence from Infosys and the Indian Software Industry", Journal of International Business Studies.

26) Klein, A (1998), "Firm performance and board committee structure", Journal of Law and Economics, Vol 41, pp 275- 303.

27) Laczniak, G.R. and P.E. Murphy (1993), Ethical Marketing Decisions: The Higher Road, Boston, Allyn\& Bacon.

28) Liang, Neng and Joanne Li (1999). “Board Structure and Firm Performance: New Evidence from China's Private Firms", Paper presented at the Academy of Management Annual Conference, Chicago, USA, August, pp. 7-10

29) Magdi, R and R, Nadareh (2002), "Corporate governance: A framework for implementation", Britain World Group Journal, Vol 20, pp 123- 132

30) Mayur M. and Saravanan P. (2006), “Does board size really matters: an empirical investigation on Indian banking sector". ICFAI Journal of Corporate Governance, Vol. 5, No. 2.

31) MohantyPitibas (2003), "Institutional Investors and Corporate Governance in India" conference paper at fifteenth AIMS conference held at XIM Bhubaneswar.

32) Narasimhan M S and JaiswallManju( 2007), "Role and Functioning of Remuneration Committee in Improving Corporate
Governance", The IUP Journal of Corporate Governance, July.

33) Parthasarathy, A., Menon, $K$ and Bhattacharjee, D. (2006). "Executive compensation, firm Performance and corporate governance: An empirical analysis. Economic and Political Weekly", 41: pp.4139-47.

34) Patibandla, M. (2006), "Equity Pattern, Corporate Governance and Performance," Journal of Economic Behavior and Organization, Vol 59, pp. 29-44.

35) Pearce, J. A., and S. A. Zahra. (1992), "Board Composition from a Strategic Contingency Perspective." Journal of Management Studies, 29,pp. 411-438

36) Phani, B. V., Reddy, V. N., Ramachandran, N. and Bhattacharya, Asish, K. (2005), "Insider Ownership, Corporate Governance and Corporate Performance", NSE Research Initiative Proposal NO.89 (www.ssrn.com), accessed on 16 April, 2013

37) Post, J.E., Preston, L.E. and Sachs, S. (2002), Redefining the Corporation: stakeholder Management and Organizational Wealth, Stanford University Press, Stanford, CA.

38) Prasanna, P. Krishna, Corporate Governance (2006), "Independent Directors and Financial Performance: An Empirical Analysis", Indian Institute of Capital Markets 9th Capital Markets Conference Paper.

39) Ramaswamy, K., Veliyath, R., Gomes, L. (2000), "A study of the determinants of CEO compensation in India", Management International Review 40(2), pp.167-191.

40) Rappaport, A. (1986), Creating Shareholder Value, the New Standard of Business Performance, The Free Press, New York, NY.

41) Sanda, A.U, A.S, Mikailu and T, Garba, (2005), "Corporate governance mechanisms and firm financial performance in Nigeria", AERC Research Paper 149, Nairobi

42) Sanda, A.U, A.S, Mikailu and T, Garba, (2005), "Corporate governance mechanisms and firm financial performance in Nigeria", AERC Research Paper 149, Nairobi. 
43) Shleifer, A, and R.W, Vishny (1997), "A survey of corporate governance", Journal of Financial Economics, Vo. 52(2), pp 737- 783.

44) SarkarJayati and Sarkar S. (2000)," Large Shareholder Activism in Developing Countries: Evidence from India," International Review of Finance, September.

45) Steward, G. (1991), The Quest for Value, Harper Collins, New York, NY

46) Uche, C (2004), "Corporate governance in Nigerian financial industry", Chartered Institute of Bankers of Nigeria Journal, Vol. 2, pp.11- 23.

47) Varma, J. (1997), "Corporate Governance in India: Disciplining the Dominant Shareholder", IIMB Management Review, Oct- Dec., 9 (4), pp. 5-18.

48) Weisbach, M (1988), “Outside directors and CEO turnover", Journal of Financial Economics, Vol. 20, pp 431- 460.

49) Wolfensohn, (1999), “Corporate governance is about promoting corporate fairness, transparency and accountability", Financial Times, 21st June, 1999.

50) Yermack, David(1996), Higher market valuation of companies a small board of directors, Journal of Financial Economics 40, pp.185-202. 